\title{
Impedance Model-Based SSR Analysis for TCSC Compensated Type-3 Wind Energy Delivery Systems
}

Lakshan Piyasinghe, Zhixin Miao, Senior Member, IEEE, Javad Khazaei, and Lingling Fan, Senior Member, IEEE

\begin{abstract}
This paper employs impedance-model-based frequency domain analysis to detect subsynchronous resonances (SSR) in Type 3 wind farms with Thyristor Controlled Series Capacitor (TCSC). The contributions of this paper are (i) the derivation of dynamic phasor based TCSC impedance model and (ii) the application of such impedance model in Type 3 wind energy systems for SSR analysis. Impedance models for TCSC with constant firing angle control and impedance control are derived in this paper. With the derived impedance models, Nyquist-stabilitycriterion is applied to compare SSR stability in Type 3 wind farm with TCSC or with fixed capacitor compensation. This paper employs analytical models to demonstrate TCSC's capability in avoiding SSR in Type 3 wind generator interconnection systems. The analytical results obtained through impedance models are validated by detail model-based (with thyristor switch modeled) time-domain simulation in Matlab/SimPowerSystems.
\end{abstract}

Index Terms-Dynamic Phasor, Impedance Model, Thyristor Controlled Series Compensator (TCSC), Sub Synchronous Resonance (SSR), Doubly Fed Induction Generator (DFIG)

\section{INTRODUCTION}

$\mathbf{U}$ TILITY industry has been concerned with SSR issues in Type 3 wind generator with series compensated network [1]. The authors have published a series of papers on this topic employing eigenvalue based analysis [2]-[4] and frequency domain impedance based analysis [5]-[7]. Compared to eigenvalue based analysis where an entire system's dynamic state matrix, eigenvalues and participation factors will be examined, impedance modeling approach is a frequency domain approach. Below is a simple example of impedance based stability analysis. The current in the system presented

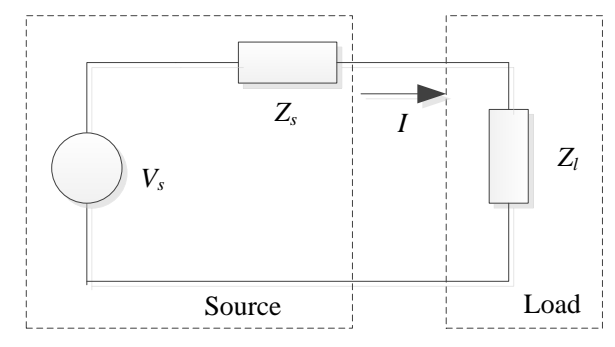

Fig. 1. Voltage source and a load.

in Fig. 1 can be written as

$$
I(s)=\frac{V(s)}{Z_{s}(s)+Z_{l}(s)}=\frac{V(s)}{Z_{l}(s)} \frac{1}{1+\frac{Z_{s}(s)}{Z_{l}(s)}} .
$$

L. Piyasinghe, Z. Miao, J. Khazaei and L. Fan are with the Department of Electrical Engineering, University of South Florida, Tampa, FL 33620. Email: linglinfan@usf.edu.
According to [8], for the stability analysis it has been assumed that the source voltage is stable when it is unloaded and the load current is also stable when powered from an ideal source. This is the same as both $V_{s}(s)$ and $\frac{1}{Z_{L}(s)}$ are stable such that stability of the current $\left(I_{s}(s)\right)$ depends on stability of second term in (1). Naturally, a circuit analysis problem now converts to a feedback control problem and the stability of the system can be judged by the loop gain $\frac{Z_{s}(s)}{Z_{l}(s)}$. Nyquist stability criterion can be applied to detect instability for this feedback control system.

Impedance modeling also provides a modular approach. We can develop an impedance model for a doubly fed induction generator (DFIG) and an impedance model for a transmission line. To examine the impact of line parameters on SSR, we only need to concern the line impedance model. Therefore, impedance modeling approach can provide insights into investigation of resonance stability. While our previous research focuses on systems with fixed capacitor compensation, this paper will examine the effect of TCSC on SSR in Type 3 wind energy systems. It has been claimed in the literature that TCSC is neutral to SSR [9]. In addition, Varma et al have published experimental results to demonstrate that TCSC can successfully suppress SSR in Type 1 wind generator systems [10]. The purpose of this paper is to provide a theoretic explanation to show why TCSC has such capability. The approach we adopt is frequency domain impedance modeling and analysis.

TCSC is difficult to model due to its low-order harmonic components in inductor currents and capacitor voltages. In the literature, other than dynamic phasor based models, TCSC's small-signal model has been developed by three approaches. In the first approach [11], frequency responses in Bode plots are obtained from small perturbation of time-domain simulation models. From these Bode plots, transfer functions are identified. Such method is also called frequency scanning and has been employed in HVDC models development [12] and SSR studies [13] and [14]. The first approach relies on

experiments and does not give analytical models. Such models can be obtained in spite of the system complexity. However, the disadvantages for system identification approach are listed as follows.

i There can be many possibilities of model structures and order. Without a prior knowledge of the TCSC model, it 
is difficult to first assume the structure of the model;

ii In general, system identification approach has the following weakness. Some parameters are not sensitive to the input/output measurements and therefore are not detectable. Many a times, we cannot directly relate a parameter with physical meaning (e.g., the gain of the firing angle PI controller) to the coefficients in an identified transfer function.

In the second approach, TCSC's steady-state model is derived [15] based on Fourier analysis and can be expressed in terms of firing angle. This model can be used to develop an approximate impedance model. The second approach does not provide a dynamic model.

In the third approach, TCSC's analytical model is derived based on discrete time state space approach [16]-[21]. The analytical models based on discrete time state space approach not only predict the system behavior precisely, but also considered as good analytical basis for control design. However, they have some disadvantages such as: complicated modeling derivations; lack of clear relation between the system configuration and the model structure, and lack of interfacing capability between the model and standard phasor-based models of generator dynamics [22].

In this paper, dynamic phasor-based modeling approach will be used to develop impedance model for TCSC. Dynamic phasor based modeling technique can include harmonic and unbalance effects in analytical models and has been employed in the past for time-domain simulation and smallsignal analysis [22]. The contribution of this paper is to employ dynamic phasor technique in deriving accurate impedance models for TCSC with constant firing angle control and constant impedance control. With the derived impedance models, Nyquist-stability-criterion can be applied to detect SSR stability in systems with TCSC. This paper successfully demonstrates TCSC's capability in avoiding SSR in Type 3 wind generator interconnection systems. In addition, effect of DFIG rotor side converter current control on SSR stability is also investigated. The analytical results obtained through impedance models are validated by detail model based time-domain simulation in Matlab/SimPowerSystems.

The paper is organized as follows. Following Section I Introduction, Section II presents dynamic phasor concept and Section III presents modeling and the derivation of TCSC impedance models under constant firing angle and constant impedance control. Frequency-domain analysis is presented in Section IV. Section V presents simulation results validating the analysis results. Section VI concludes this paper.

\section{DyNAMIC Phasor CONCEPT}

The Fourier series representation of a complex time domain waveform $x(\tau)$ in the interval $\tau \in(\tau-T, t)$ can be presented as

$$
x(\tau)=\sum_{k=-\infty}^{\infty} X_{k}(t) \cdot e^{j k \omega \tau}
$$

Here $\omega=\frac{2 \pi}{T}$ and $X_{k}(t)$ is the $k^{t h}$ complex Fourier coefficient. The coefficients are also referred as dynamic phasor coefficients which can be obtained using the following average operation [22].

$$
X_{k}(t)=\frac{1}{T} \int_{t-T}^{t} x(\tau) e^{j k \omega \tau} \mathrm{d} \tau=\langle x\rangle_{k}(t)
$$

Dynamic phasor $X_{k}$ is also notated as $\langle x\rangle_{k}$, where $\langle.\rangle_{k}$ represents the dynamic phasor of the $k$-th harmonic component.

One of the most important properties of dynamic phasor is the relationship between derivative of original signal and the derivative of dynamic coefficient, which can be obtained using (2).

$$
\left\langle\frac{d x}{d t}\right\rangle_{k}=\frac{d X_{k}}{d t}+j k \omega X_{k}
$$

Considering only the fundamental frequency $\left(\omega_{s}\right)$ component, the dynamic phasor based impedance models for a series RL circuit and a capacitor can be derived from the RL circuit dynamics expressed in (5).

$$
\begin{aligned}
& R i+L \frac{d i}{d t}=v \\
\Rightarrow & R\langle i\rangle_{1}+L\left(\frac{d\langle i\rangle_{1}}{d t}+j \omega_{s}\langle i\rangle_{1}\right)=\langle v\rangle_{1}
\end{aligned}
$$

where $i$ is the instantaneous current through the resistor with resistance $R$ and the inductor with inductance $L$ and $v$ is the instantaneous voltage across the RL circuit terminal.

Applying Laplace transformation, the ratio of the voltage phasor against the current phasor in frequency domain can be found:

$$
\frac{\langle v\rangle_{1}}{\langle i\rangle_{1}}=R+\left(s+j \omega_{s}\right) L .
$$

This impedance is in the complex domain. In the real domain, an impedance matrix is defined as

$$
\left[\begin{array}{c}
\langle v\rangle_{1}^{R} \\
\langle v\rangle_{1}^{I}
\end{array}\right]=Z\left[\begin{array}{c}
\langle i\rangle_{1}^{R} \\
\langle i\rangle_{1}^{I}
\end{array}\right]
$$

where the superscripts $R$ and $I$ refer the real and imaginary parts of a complex variable and $V_{1}=V_{1}^{R}-j V_{1}^{I}$.

For a transmission line modeled as a series RL circuit, the impedance model is expressed as (7). Similarly, for a capacitor $C$, the impedance model is expressed as (8).

$$
\begin{aligned}
Z_{\text {line }} & =\left[\begin{array}{cc}
R+s L & \omega_{s} L \\
-\omega_{s} L & R+s L
\end{array}\right] \\
Z_{F C} & =\left[\begin{array}{ll}
\frac{s}{\left(s^{2}+\omega_{s}^{2}\right) C} & -\frac{\omega_{s}}{\left(s^{2}+\omega_{s}^{2}\right) C} \\
\frac{\omega_{s}}{\left(s^{2}+\omega_{s}^{2}\right) C} & \frac{s}{\left(s^{2}+\omega_{s}^{2}\right) C}
\end{array}\right]
\end{aligned}
$$

\section{IMPEDANCE MODEL OF TCSC}

With the assumption of a sinusoidal imposed voltage, the fundamental frequency current through a Thyristor Controlled Reactor (TCR) can be obtained and further the inductance can be expressed as [23]:

$$
L(\alpha)=L \frac{\pi}{\pi-2 \alpha-\sin (2 \alpha)}
$$

where $\alpha$ is the firing angle measured from the zero crossing of the line current and $L$ is the inductance of the thyristorcontrolled reactor. 


$$
\begin{aligned}
& \underbrace{\left[\begin{array}{c}
V_{1}^{R} \\
\dot{V_{1}^{I}} \\
\dot{I_{1}^{R}} \\
\dot{I_{1}^{I}}
\end{array}\right]}_{\dot{X}}=\underbrace{\left[\begin{array}{cccc}
-\omega_{s} & -\frac{1}{C} & 0 \\
\omega_{s} & 0 & 0 & -\frac{1}{C} \\
\frac{1}{\pi L}[\sigma+\sin (\sigma) \cos (2(\xi+\phi))] & \frac{1}{\pi L} \sin (\sigma) \sin (2(\xi+\phi)) & 0 & -\omega_{s} \\
\frac{1}{\pi L} \sin (\sigma) \sin (2(\xi+\phi)) & \frac{1}{\pi L}[\sigma-\sin (\sigma) \cos (2(\xi+\phi))] & \omega_{s} & 0
\end{array}\right]}_{A} \underbrace{\left[\begin{array}{c}
V_{1}^{R} \\
V_{1}^{I} \\
I_{1}^{R} \\
I_{1}^{I}
\end{array}\right]}_{X} \underbrace{\left[\begin{array}{c}
\frac{1}{C} \\
0 \\
0 \\
0 \\
0 \\
0 \\
0 \\
0 \\
0
\end{array}\right]}_{B} \underbrace{\left[\begin{array}{c}
I_{l 1}^{R} \\
I_{l 1}^{I}
\end{array}\right]}_{U} \\
& \underbrace{\left[\begin{array}{c}
V_{1}^{R} \\
V_{1}^{I}
\end{array}\right]}_{Y}=\underbrace{\left[\begin{array}{llll}
1 & 0 & 0 & 0 \\
0 & 1 & 0 & 0
\end{array}\right]}_{C} \underbrace{\left[\begin{array}{c}
V_{1}^{R} \\
V_{1}^{I} \\
I_{1}^{R} \\
I_{1}^{I}
\end{array}\right]}_{X}
\end{aligned}
$$

Therefore, a simple TCSC impedance model per-phase can be expressed as:

$$
Z_{T C S C}(s)=\frac{s L(\alpha)}{1+s^{2} L(\alpha) C}
$$

The assumption of undistorted voltage is not the case in TCSC. Instead, undistorted line current assumption is usually used for TCSC. Jalali et al [15] derived a complex steady-state reactance model for a TCSC. To account for dynamics and develop frequency domain impedance model, we start from the state-space model of fundamental frequency component developed in [22].

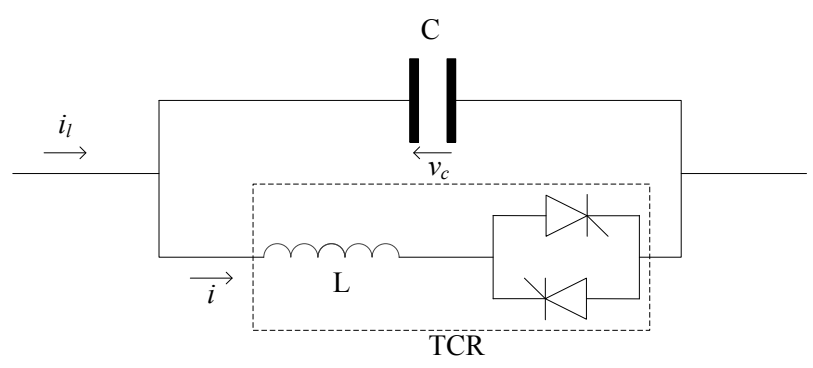

Fig. 2. TCSC circuit diagram.

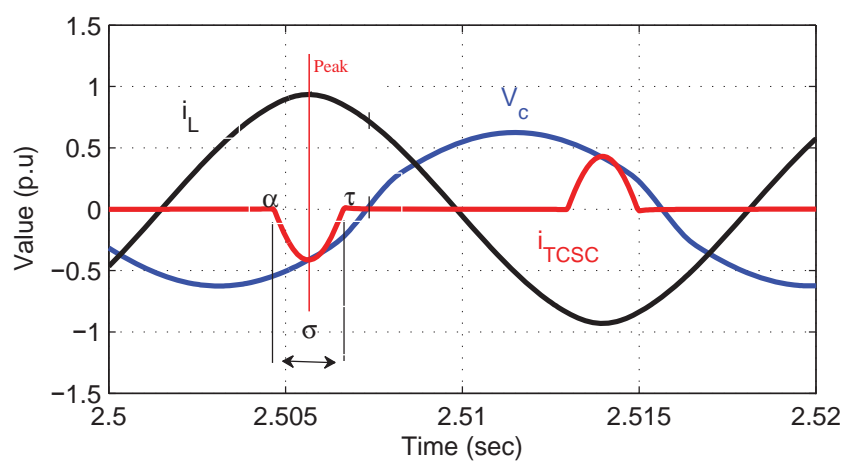

Fig. 3. TCSC waveforms.

The circuit diagram of a TCSC is presented in Fig .2. This circuit consists of a capacitor in parallel with a Thyristor Controlled Reactor (TCR). The net impedance of the TCSC can be controlled via controlling the fire angle $\alpha$ of the TCR. Fig. 3 shows the steady state voltage and current waveforms of the TCSC. The Thyristor is switched on $\alpha$ angle after the zero crossing of the line current and will be conducting till $\tau$. Conduction angle is defined as, $\sigma=\tau-\alpha$ and it can be assumed that the angle $\sigma$ is symmetrical with respect to the peak value of the line current to simplify model development. This assumption is employed in [22]. The dynamic phasor model for the fundamental voltage and current phasors can be developed as follows.

The dynamics of the TCSC in phase domain are as follows:

$$
\left\{\begin{array}{l}
C \frac{d v_{c}}{d t}=i_{l}-i \\
L \frac{d i}{d t}=q v_{c}
\end{array}\right.
$$

where $i_{l}$ is the line current $v_{c}$ is the voltage across the capacitor, $i$ is the current through the TCR and $q$ is the switching function which represents the TCR switching. $q=1$ when one of the thyristors is conducting and $q=0$ when both are not conducting. Fundamental dynamic phasor representation of (13) can be obtained using the dynamic phasor concept.

$$
\begin{cases}C \frac{d V_{1}}{d t} & =I_{l 1}-I_{1}-j \omega_{s} C V_{1} \\ L \frac{d I_{1}}{d t} & =\left\langle q v_{c}\right\rangle_{1}-j \omega_{s} L I_{1}\end{cases}
$$

where $\langle.\rangle_{k}$ represents the dynamic phasor of the $k$-th harmonic component, subscript " 1 " denotes phasors related to the fundamental frequency. These phasors are complex variables and can be expressed by the real part and the imaginary part.

$$
\left\{\begin{array}{l}
V_{1}=V_{1}^{R}-j V_{1}^{I} \\
I_{1}=I_{1}^{R}-j I_{1}^{I} \\
I_{l 1}=I_{l 1}^{R}-j I_{l 1}^{I}
\end{array}\right.
$$

When a TCSC is operating in capacitive region, the fundamental component gives a good approximation for the capacitor voltage, $v_{c}$ [22]. Hence $v_{c}$ can be represented by only fundamental dynamic phasor coefficients,

$$
v_{c}=V_{1} e^{j \omega_{s} t}+V_{1}^{*} e^{-j \omega_{s} t}
$$

Assuming the fundamental component of the inductor current is symmetric with respect to the peak of actual inductor current, which means both peaks will happen at the same time as it can be observed in Fig. 3, then 


$$
\left\langle q v_{c}\right\rangle_{1}=\frac{2}{\pi} \int_{\alpha}^{\tau} v_{c} e^{-j \theta} d \theta=\frac{1}{\pi}\left[V_{1} \sigma+V_{1}^{*} \sin (\sigma) e^{-2 j(\xi+\phi)}\right]
$$

where the definitions of $\xi$ is the phase angle corresponding to the peak value of the line current and $\phi$ is the phase shift between line current $I_{L 1}$ and the fundamental component of current $I_{1}$ through TCR [22]:

$$
\begin{aligned}
\xi & =\arg \left(I_{L 1}^{*}\right) \\
\phi & =\arg \left(-I_{L 1} \cdot I_{1}^{*}\right)
\end{aligned}
$$

At steady state, $\xi$ is considered to be $\frac{\pi}{2}$ and $\phi$ is considered as 0 . Separating the real and imaginary parts of dynamic

phasors, a fourth-order state-space model of TCSC can be obtained. The system model is presented in (9) and (10).

1) Impedance Model with Fixed $\alpha$ : With fixed $\alpha$ control, the system matrix $A$ is a constant matrix, hence the state space model given in (9) and (10) can be utilized to obtain the impedance matrix directly as follows.

$$
Z_{T C S C}(s)=\frac{Y(s)}{U(s)}=C(s I-A)^{-1} B .
$$

2) Impedance Model with $\alpha$ Control : Fig. 5 shows the impedance control loop of the TCSC. The unit of impedance is $\Omega$ and the unit of $\alpha$ is degree. Therefore, the unit of $K_{p}$ is degree/ $\Omega$ and the unit of $K_{i}$ is degree.s $/ \Omega$. The Laplace expression of the firing angle is as follows.

$$
\Delta \alpha(s)=-H(s) \Delta Z(s) .
$$

Impedance $Z$ is computed from the instantaneous voltage and current measurements as shown in Fig. 5. The expression of $Z$ is as follows:

$$
Z=\left|\frac{V_{1}}{I_{l 1}}\right|=\sqrt{\frac{\left(V_{1}^{R}\right)^{2}+\left(V_{1}^{I}\right)^{2}}{\left(I_{l 1}^{R}\right)^{2}+\left(I_{l 1}^{I}\right)^{2}}}
$$

where $I_{l}^{0}$ and $V_{1}^{0}$ are the fundamental components of initial line current and capacitor voltage.

Applying small perturbation and the resulting impedance deviation $\Delta Z$ can be expressed as:

$$
\Delta Z=\left[\frac{\partial Z}{\partial X}\right]^{T} \Delta X+\left[\frac{\partial Z}{\partial U}\right]^{T} \Delta U
$$

When $\alpha$ is controlled, the system matrix $A$ is no longer a constant matrix. The resulting small-signal state-space model is given as:

$$
\Delta \dot{X}=A\left(\alpha_{0}\right) \Delta X+\frac{\partial A}{\partial \alpha} X_{0} \Delta \alpha+B \Delta U
$$

Applying Laplace transformation leads to

$$
s \Delta X(s)=A\left(\alpha_{0}\right) \Delta X(s)+\frac{\partial A}{\partial \alpha} X_{0} \Delta \alpha+B \Delta U(s) .
$$

Substituting (20) and (22) into (24) leads to

$$
\begin{aligned}
& \frac{\Delta X(s)}{\Delta U(s)}= \\
& \underbrace{\left(s I-A+\frac{\partial A}{\partial \alpha} X_{0} H(s) \frac{\partial Z}{\partial X}\right)^{-1}\left(-\frac{\partial A}{\partial \alpha} X_{0} H(s) \frac{\partial Z}{\partial U}+B\right)}_{G_{U X}(s)} .
\end{aligned}
$$

Hence the impedance model is expressed as:

$$
Z_{T C S C}(s)=\frac{\Delta Y(s)}{\Delta U(s)}=C G_{U X}(s)
$$

\section{FREQUENCY DOMAIN ANALYSis}

\section{A. Stability Criterion}

For single-input single-output (SISO) systems, Nyquist plots and Bode plots of the loop gain $Y(s) Z(s)$ can be used to detect stability issues and determine phase margin and gain margins. However, the derived impedance models in this paper are two by two matrices. In turn, the equivalent control systems are multi-input multi-output (MIMO) systems. To examine stability for MIMO systems, [24] proposes to plot the Nyquist maps of the eigenvalues of the loop gain. Such technique is employed in [25].

Singular values of the return matrix $T(s)=I+$ $Y_{l}(s) Z_{s}(s)$ delivers a good measure of stability of a system since the minimum singular value of the return matrix is equivalent to the distance between the Nyquist locus and the critical point $(-1,0)$ in the SISO case [26], [27]. The difference between maximum and minimum singular value can be used as a indicator of the system "ill conditioning" [26]. The larger the difference, the more the system is prone to "ill condition".

According to [27], if the return difference matrix $T(s)$ is nearly singular, then there exists a small perturbation in $Y_{l}(s) Z_{s}(s)$ that will destabilize the closed-loop system. The minimum singular value of $T(s)$ measures the nearness to singularity of $T(s)$. The minimum singular value approach is useful in detecting the near instability, it is unnecessarily conservative. This is due to the fact that some of the small perturbations that would destabilize the closed-loop system will never occur in the physical system.

It can be further expressed as, if $\sigma_{\min }[T(j \omega)]$ represents the smallest singular value at the frequency $\omega$, and if there is a constant $\beta \leq 1$ such that $\sigma_{\min }[T(j \omega)] \geq \beta$ for all frequencies, then there is a guaranteed gain margin and phase margin of [26], [27].

$$
\begin{aligned}
& G M=\frac{1}{1 \pm \beta} \\
& P M= \pm \cos ^{-1}\left(1-\frac{\beta^{2}}{2}\right)
\end{aligned}
$$

Hence, if the minimum singular value of the return reference matrix is equal to or more than 1 , the system is guaranteed to have a phase margin of \pm 60 which means the system is stable.

On the other hand, if the minimum singular value of the return reference matrix is less than 1 , the system is seen as not to have sufficient stability margin and the system is prone to resonances. The corresponding frequency at the minimum singular value indicates resonance frequency. This criterion ( the minimum singular value is less than 1) is used to screen out potential instable cases. . 


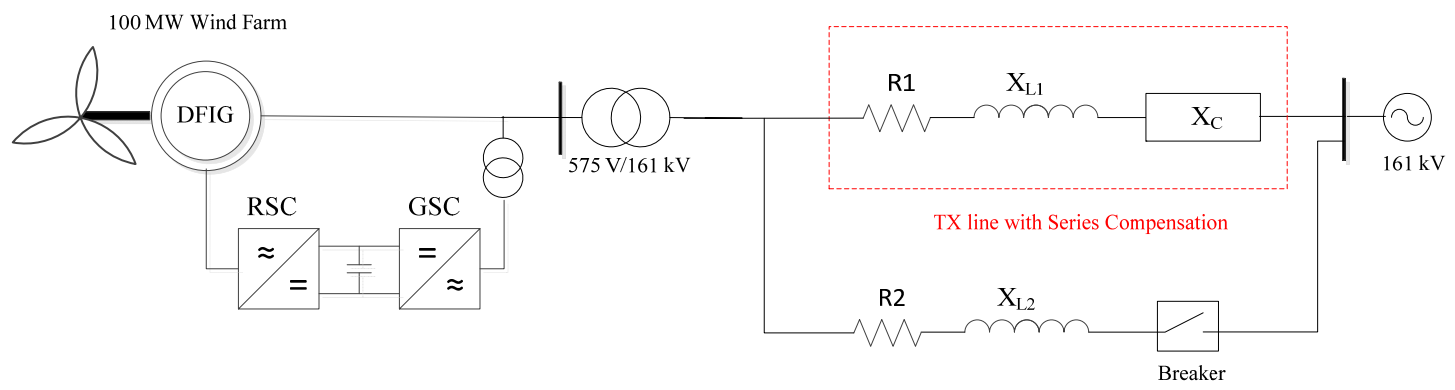

Fig. 4. The study system: a wind farm connected to grid through a series compensated transmission line.

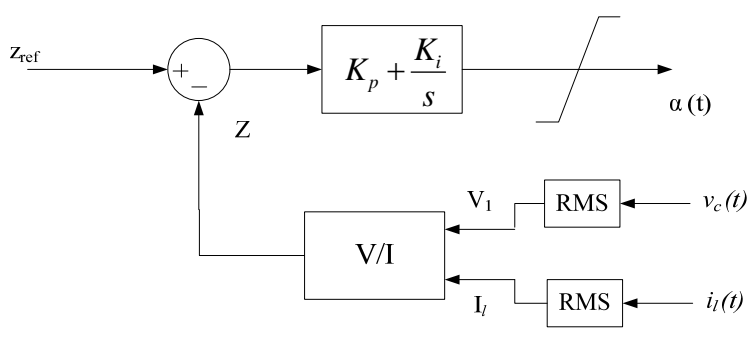

Fig. 5. Control block diagram for $\alpha$ Control in TCSC.

\section{B. Case study}

In this subsection, the developed TCSC impedance model is used for stability analysis. A case study considered is a Type 3 wind farm of 100 MW interconnected with a series compensated network at $161 \mathrm{kV}$. The system is modified from the Second Benchmark model which has been widely used for SSR studies [28]. It is composed of two parallel transmission lines. One of them is series compensated. At $t=4 \mathrm{~s}$, the three-phase breaker on the non-compensated line will be tripped. The wind farm is now radially connected to a series compensated network. The system diagram is shown in Fig. 4.

The wind farm is represented with a lumped DFIG model which is connected to the system through a transformer. Case studies are carried out for two series compensation methods: fixed capacitor compensation and TCSC compensation.

DFIG's Rotor Side Converter (RSC) current controller has been found to have negative impact on SSR for fixed capacitor compensated networks [2], [6]. Therefore, in this paper, effect of RSC current controller parameters on TCSC compensated networks will be examined. The inner current control loop of the RSC is illustrated in Fig. 6. Two PI controllers are used to regulate the currents to yield a proper voltage output. The gain settings of two PI controllers are the same for $d$ - axis and $q$-axis. The unit of the currents is Ampere and the unit of the voltages are Volt. Therefore the unit of $K_{p}$ is $\Omega$ and the unit of $K_{i}$ is $\Omega / s$. The impedance model for a DFIG with RSC current control adopted in this paper comes from [6]. For fixed capacitor and TCSC scenarios, the compensation degree at $60 \mathrm{~Hz}$ will be the same. The parameters of the transmission

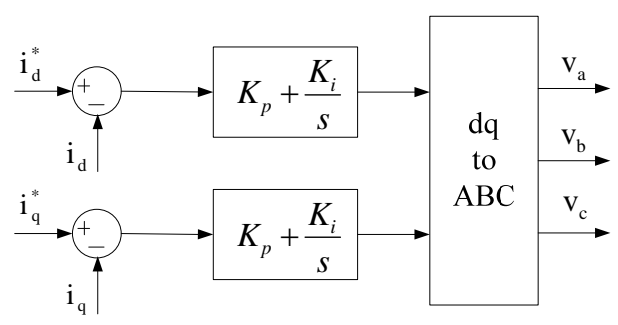

Fig. 6. Inner current control loop for Rotor Side Converter of DFIG.

line, capacitor size and TCSC parameters are listed in Table IV in Appendix.

1) Fixed Series Compensation: Phase domain based scalar impedance models for an DFIG has been developed in [6]. For a transmission line with fixed series compensation, the scalar impedance model is $R+s L+\frac{1}{s C}$.

Figs. 7 and 8 present the Nyquist plot for the loop gain $Y_{D F I G}(s) Z_{n e t}(s)$ (The loop gain is a scalar since both the DFIG and the compensated line are modeled in phase domain.) and the Bode plots for $Z_{D I F G}(s)$ and $Z_{n e t}(s)$, where $Z_{n e t}(s)$ is the impedance of the series compensated line, $Z_{D I F G}(s)$ is the DFIG impedance, and $Y_{D F I G}=Z_{D F I G}^{-1}$. The two figures show the resonance frequencies at different compensation levels. Observed from the Bode plots, the higher the compensation degree, the network resonance frequency $f_{n}$ will be greater. Resonance frequencies for $40 \%, 50 \%$, and 70 $\%$ are $29 \mathrm{~Hz}, 32 \mathrm{~Hz}$ and $38 \mathrm{~Hz}$, respectively. It is shown that phase margin reduced with the increase of the compensation level. Nyquist plots for all compensation levels encircle $[-1,0]$ in clockwise directions, which implies that the system goes unstable for all compensation levels. SimPowersystems simulation presented in Section V will confirm instability for this scenario. Note that the network resonant at $f_{n}$ will be observed as an oscillation mode with a complementary frequency $\left(f_{s}-f_{n}\right.$, where $f_{s}$ is $\left.60 \mathrm{~Hz}\right)$ in power.

Dynamic phasor-based matrix impedance models are also used for SSR analysis for the fixed capacitor scenario. Fig. 9 (a) presents the singular value plots of the return difference matrix $1+Y_{D F I G} Z_{n e t}$ in dynamic phasor domain. The ill conditioning frequencies marked in Fig. 9 are listed in Table I. The resonance frequencies observed from scalar impedancebased Bode plots are also listed in Table I. The two sets of the frequencies should be complement to each other due to the 


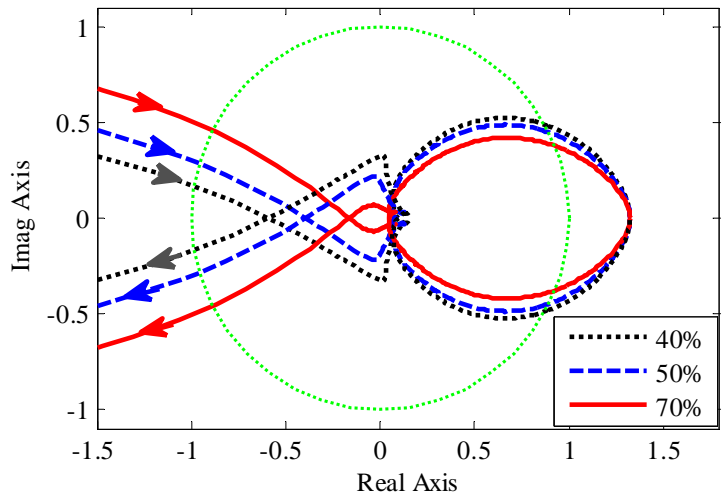

Fig. 7. Nyquist plots for fixed capacitor compensation at different compensation levels. RSC controller setting: $K_{p}=0.6, K_{i}=8$.

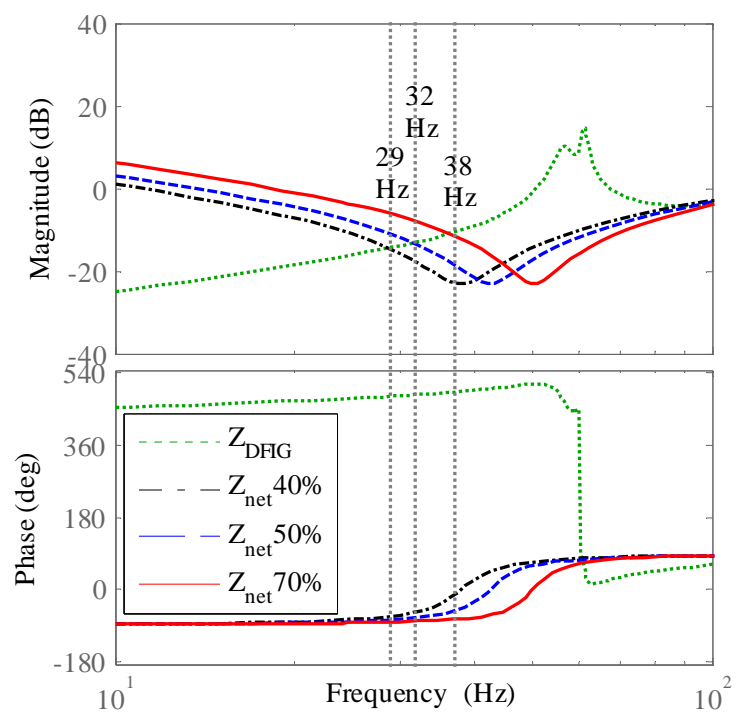

Fig. 8. Bode plots for fixed capacitor compensation at different compensation levels. RSC controller setting: $K_{p}=0.6, K_{i}=8$.

difference in reference domains. From Table I, we can see they are exactly complement to each other. Table I demonstrates that singular value plots of the return difference matrix can detect SSR. The results agree with the analysis carried out by scalar impedance based analysis.

TABLE I

RESONANCE FREQUENCIES IDENTIFIED BY PHASE DOMAIN SCALAR IMPEDANCE-BASED ANALYSIS AND DYNAMIC PHASOR DOMAIN MATRIX IMPEDANCE-BASED ANALYSIS

\begin{tabular}{|c|c|c|}
\hline Compensation & abc Phase Domain & Dynamic Phasor Domain \\
\hline \hline $40 \%$ & $29 \mathrm{~Hz}$ & $31 \mathrm{~Hz}$ \\
$50 \%$ & $32 \mathrm{~Hz}$ & $28 \mathrm{~Hz}$ \\
$70 \%$ & $38 \mathrm{~Hz}$ & $22 \mathrm{~Hz}$ \\
\hline
\end{tabular}

\section{2) TCSC effect:}

a) Fixed alpha mode: Fig. 9 (b) shows the effect of different compensation levels of TCSC in constant alpha mode on system stability. Different values for $\alpha$ are given in Table II. It can be concluded that when the TCSC is in constant alpha mode, the system has no stability issues for all the compensation levels because the absolute values of the singular values are greater than one for all three cases.

TABLE II

COMPENSATION LEVEL, FIRING ANGLE AND EQUIVALENT IMPEDANCE AT FUNDAMENTAL FREQUENCY OF TCSC

\begin{tabular}{|c|c|c|}
\hline Compensation & $\alpha$ & $Z_{\text {ref }}$ \\
\hline \hline $40 \%$ & $75^{0}$ & $52 \Omega$ \\
$50 \%$ & $71.9^{0}$ & $65 \Omega$ \\
$70 \%$ & $69.35^{0}$ & $91 \Omega$ \\
\hline
\end{tabular}

b) Impedance control mode: Effect of different compensation levels when the TCSC is operated with impedance control in capacitive mode is illustrated in Fig. 9 (c). The reference values of TCSC impedances for different compensation levels are presented in Table II. Fig. 9 (c) shows that as the compensation level increases to $70 \%$, the system lacks stability margin and may be unstable and the frequency of the potential resonance is $22 \mathrm{~Hz}$. The system is stable for $40 \%$ and $50 \%$ compensation.

c) Effect of RSC current control: In this case, the TCSC is in impedance control (capacitive mode). The DFIG RSC current controller parameters are varied. Fig. 10 presents the singular values of the return difference matrix. It is found that when the TCSC is in constant impedance control mode, for the selected RSC gains, the system is stable. This analysis results demonstrate the significant improvement on SSR stability due to TCSC. [2] and [6] have shown that RSC current control contributes to SSR instability when fixed capacitor is used for series compensation and increasing the gains of RSC current control aggravates instability. Analysis in this paper shows that for TCSC compensated wind farms, RSC current control no longer affects SSR.

\section{Time-Domain Simulation Results}

In this section, the analysis results will be verified by the time-domain simulation results. The study system is constructed in Matlab/SimPowersystems. The DFIG model and the TCSC model are from SimPowersystems library. The TCSC simulation model includes thyristor switches and TCSC control dynamics. The detailed model of DFIG for simulation studies consists of the following elements:

1) power converter IGBT bridges and pulse width modulation (PWM) blocks;

2) RSC and GSC inner current loops and outer power/voltage loops;

3) RSC and GSC phase-locked loops (PLLs).

Two scenarios will be studied.

\section{A. Effect of different fixed capacitor compensation level}

In this case study, a fixed capacitor is employed to provide series compensation in one of the transmission lines. A line trip is considered for the uncompensated $\mathrm{AC}$ line at $t=4 \mathrm{~s}$. Results of simulation for different compensation level are illustrated in Fig. 11. It can be observed that, as the compensation level increases, the system is more prone to instability. For 


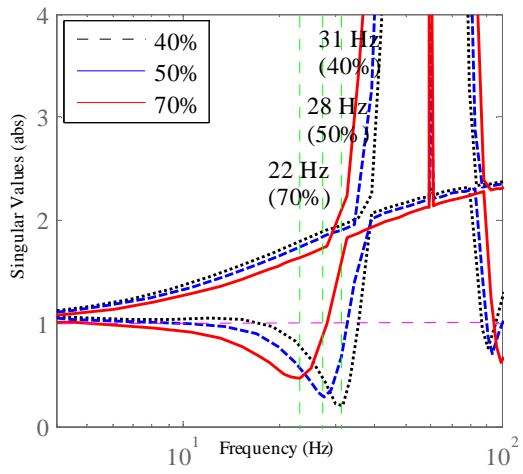

(a)

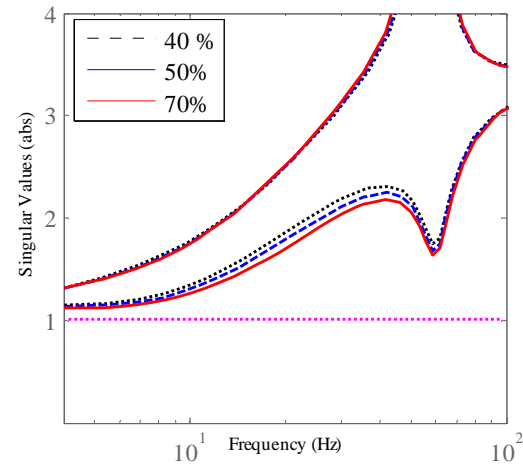

(b)

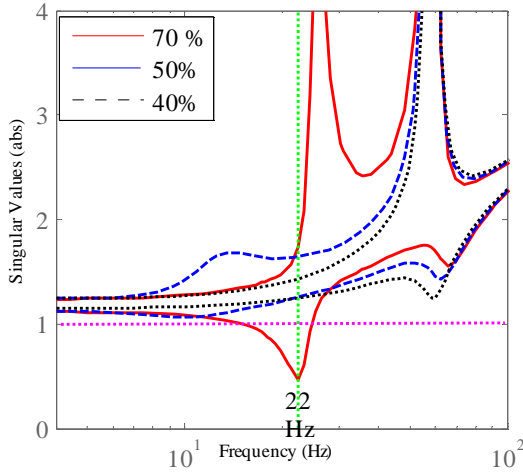

(c)

Fig. 9. Singular value plots of $1+Y_{D F I G} Z_{\text {net }}$ for different compensation levels. (a) Fixed Capacitor; (b) Constant alpha mode for TCSC; (c) Impedance control of TCSC. RSC current control parameters: $K_{P}=0.6, K_{i}=8$, TCSC controller parameters: $K_{P}=0.53, K_{i}=3$

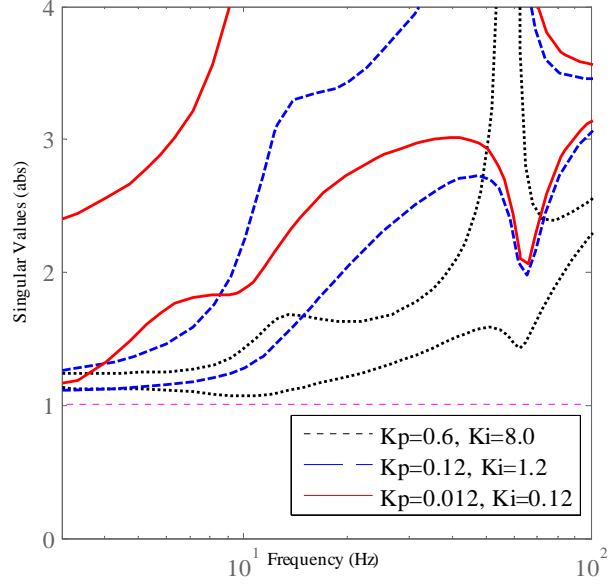

Fig. 10. Singular value plot of $1+Y_{D F I G} Z_{n e t}$ for different $K_{p}, K_{i}$ of RSC. TCSC reference impedance is set at $50 \%$ compensation level. TCSC control parameters: $K_{P}=0.32, K_{i}=1.84$.

the all the compensation levels, the system loses stability. The dominant mode in this condition has a frequency about 21 $\mathrm{Hz}$ for $70 \%$ compensation, $28 \mathrm{~Hz}$ for $50 \%$ compensation and $32 \mathrm{~Hz}$ for $40 \%$ compensation. The simulation results are in agreement with the frequency domain analysis.

\section{B. Effect of fixed alpha mode of TCSC}

For the constant firing angle of TCSC, the analysis has shown that the system will be stable even with $70 \%$ compensated system. As the system is stable for all the compensation levels, simulation result for constant alpha mode under $70 \%$ compensation of transmission line has been included in Fig. 12. It can be observed that the system is stable after a fault. This is in agreement with the analysis results given in Fig. 9 (b).

\section{Comparison of TCSC and Fixed Capacitor}

In this case study, the compensation level is selected as $70 \%$. Two scenarios, one using TCSC for series compensation and

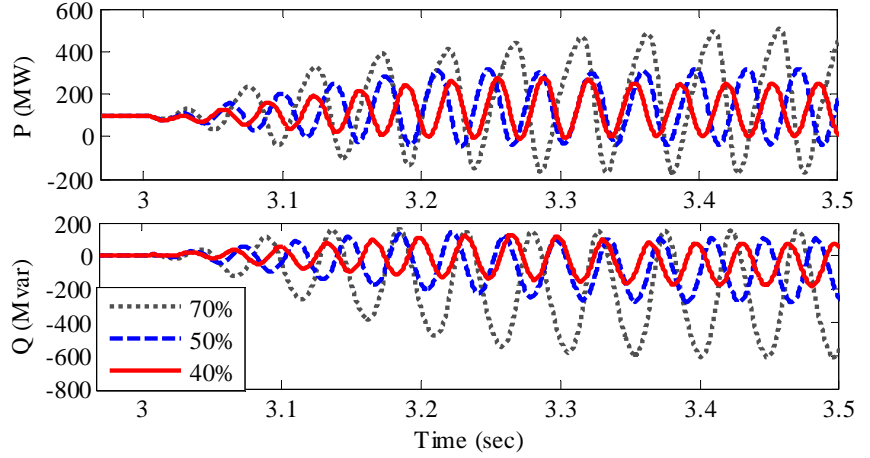

Fig. 11. Simulation results for different compensation level of Fixed Capacitor. RSC current control parameters: $K_{P}=0.6, K_{i}=8$.

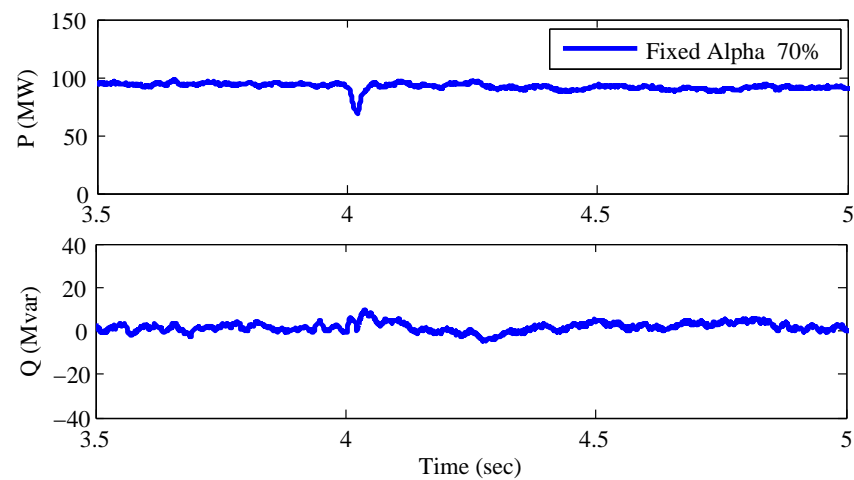

Fig. 12. Simlulation results for constant alpha mode when three phase fault happens at $4 \mathrm{sec}$ for 2 cycles and compensation level is set to $70 \%$.

the other using fixed capacitor for series compensation, are compared. The simulation results are presented in Fig. 13. It can be observed that, as the line trip happens, the fixed capacitor compensated power system fails to sustain stability and large fluctuations will be experienced in the active and reactive power. In contrast, the TCSC in impedance control mode can mitigate SSR and the system will retain the stability after a few cycles. The TCSC control parameters are selected 
to be low to have robust performance. Indicated in the next case study, if the TCSC control parameters are large, SSR could appear at $70 \%$ compensation level.

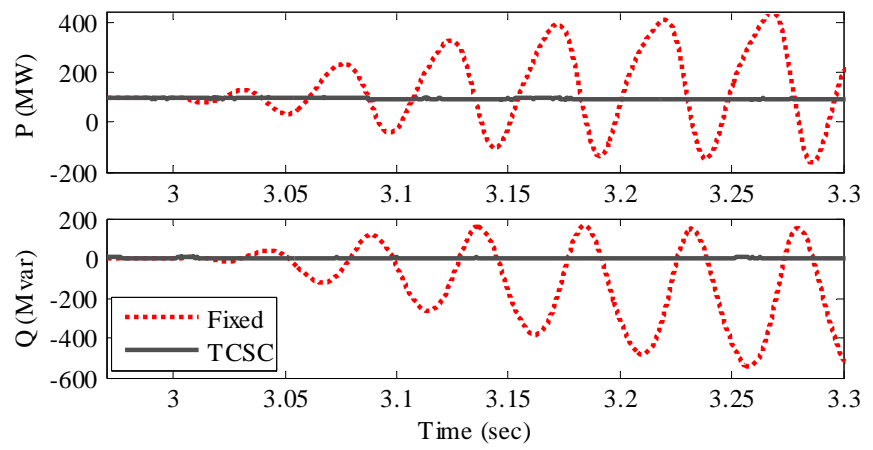

Fig. 13. Results of comparison between Fixed Capacitor and TCSC in 70\% compensation of the line reactance. RSC: $K_{P}=0.6, K_{i}=8$,TCSC: $K_{P}=$ $0.16, K_{i}=0.92$

\section{Effect of different TCSC compensation level}

In this case study, a TCSC is employed to provide series compensation in one of the transmission lines. A line trip is considered for the uncompensated $\mathrm{AC}$ line at $t=4 \mathrm{~s}$. Three different compensation levels have been considered for TCSC at $40 \%, 50 \%$, and $70 \%$ in impedance control mode. Simulation results are presented in Fig. 14. It can be observed that when the compensation level is increased to $70 \%$, the active power of the line is experiencing oscillations around $22 \mathrm{~Hz}$. For $40 \%$ and $50 \%$ compensation level, the system is stable. The simulation results corroborate with the singular value analysis results in Fig. 9.
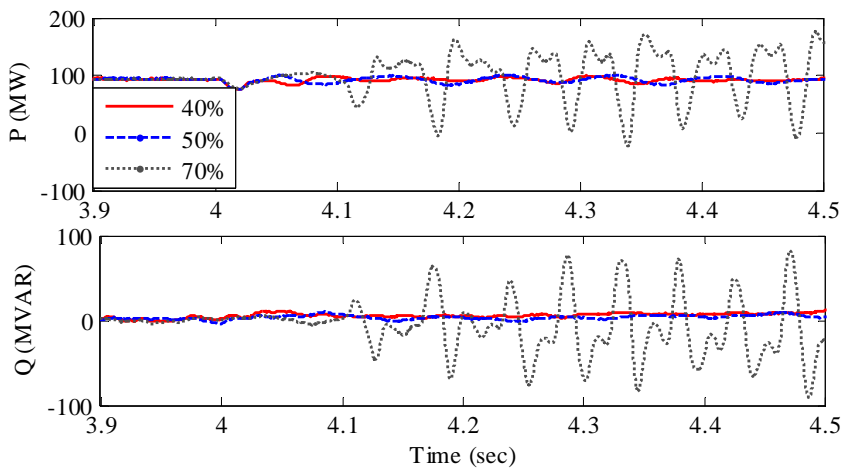

Fig. 14. Wind farm output real power and reactive power for different compensation of TCSC. RSC: $K_{P}=0.6, K_{i}=8$, TCSC gains: $K_{P}=0.53$, $K_{i}=3$.

\section{E. Effect of RSC current control on TCSC compensated system}

In this case study, RSC current controller parameters will be varied and the compensation level of the TCSC is set to $50 \%$ and the TCSC controller parameters are kept constant. All three cases are stable. The simulation results shown in Fig. 15 corroborate with the analysis results in Fig. 10. It can be observed from Fig. 15 that, as the gain settings of
RSC are increased, the system is more likely to be stable. This phenomenon is very different from the case where fixed capacitor is used. In [2], [6], studies show that increasing RSC gains makes the system prone to SSR.

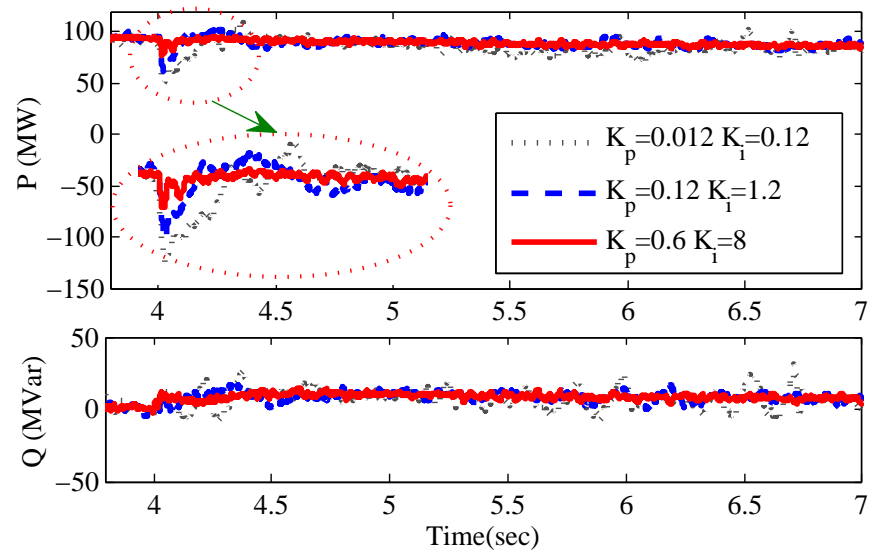

Fig. 15. Simulation results for different $K_{p}, K_{i}$ of DFIG RSC for $50 \%$ compensation level. TCSC controller paramters: $K_{P}=0.32, K_{i}=1.84$.

Remarks: The simulation results corroborate with the analysis results. Important observations are drawn as follows:

1) With a TCSC compensated transmission line, a Type3 wind farm can be radially connected with the line and operate safely given appropriate TCSC control parameters. On the other hand, if fixed capacitor-based compensation is used, Type-3 wind farms cannot be radially connected with series compensated lines due to SSR.

2) The case study demonstrates that in a TCSC interconnected Type-3 wind farm system, RSC current control does not appear to pose a threat on SSR.

\section{CONCLUSION}

This paper develops dynamic phasor based impedance models for TCSC and carries out frequency domain analysis for a system with Type 3 wind farm and TCSC. Impedance models for TCSC with constant firing angle control and impedance control are derived in this paper. With the derived impedance models, the Nyquist-stability-criterion is applied to detect SSR stability in Type 3 wind farm with TCSC. This paper employs both analysis and time-domain simulation in Matlab/SimPowersystems to demonstrate TCSC's capability in avoiding SSR in Type 3 wind generator interconnection systems. In addition, for TCSC compensation, both analysis and simulation results show DFIG RSC current control does not contribute to SSR instability. The analytical results obtained through impedance models are validated by detailed based (with thyristor switch modeled) time-domain simulation in Matlab/SimPowerSystems.

\section{APPENDIX}

\section{REFERENCES}

[1] G. Irwin, A. Jindal, and A. Isaacs, "Sub-synchronous control interactions between type 3 wind turbines and series compensated ac transmission systems," in IEEE Power \& Energy General Meeting, Jul. 2011. 
TABLE III

PARAMETERS OF THE DFIG WIND FARM

\begin{tabular}{|c|c|c|}
\hline Rated Power & $1.5 \mathrm{MW}$ & $100 \mathrm{MW}$ \\
Rated Voltage & $575 \mathrm{~V}$ & $575 \mathrm{~V}$ \\
$r_{s}(p u)$ & 0.023 & 0.023 \\
$r_{r}(p u)$ & 0.18 & 0.18 \\
$X_{l s}(p u)$ & 0.016 & 0.016 \\
$X_{l r}(p u)$ & 0.16 & 0.16 \\
$X_{M}(p u)$ & 2.9 & 2.9 \\
$H\left(k g . m^{2}\right)$ & 0.685 & 0.685 \\
$F(p u)$ & 0.01 & 0.01 \\
$p$ (poles) & 3 & 3 \\
\hline
\end{tabular}

TABLE IV

PARAMETERS OF THE TRANSMisSiOn Lines

\begin{tabular}{|c|c|}
\hline Transformer Ratio & $575 \mathrm{~V} / 161 \mathrm{kV}$ \\
Transformer $X_{t}$ & $0.1 \mathrm{pu}$ \\
Power base & $100 \mathrm{MVA}$ \\
Frequency & $60 \mathrm{~Hz}$ \\
$R_{1}$ & $0.02 \mathrm{pu}$ \\
$R_{2}$ & $0.02 \mathrm{pu}$ \\
$X_{l 1}$ & $0.5 \mathrm{pu}$ \\
$X_{l 2}$ & $0.02 \mathrm{pu}$ \\
$X_{c}$ at 50 compensation & $64.8 \Omega$ \\
$L_{T C S C}$ & $3.8 \mathrm{mH}$ \\
$C_{T C S C}$ & $65 \mathrm{e}-6 \mathrm{~F}$ \\
$\alpha$ at $50 \%$ compensation level & $72^{0}$ \\
\hline
\end{tabular}

[2] L. Fan, R. Kavasseri, Z. Miao, and C. Zhu, "Modeling of DFIG-based wind farms for SSR analysis," IEEE Trans. Power Del., vol. 25, no. 4, pp. 2073-2082, Oct. 2010.

[3] L. Fan, C. Zhu, Z. Miao, and M. Hu, "Modal analysis of a DFIG-based wind farm interfaced with a series compensated network," IEEE Trans. Energy Convers., vol. 26, no. 4, pp. 1010-1020, Dec. 2011.

[4] L. Fan and Z. Miao, "Mitigating SSR using DFIG-based wind generation," IEEE Trans. Sustainable Energy, vol. 3, no. 3, pp. 349-358, Jul. 2012.

[5] — - "Nyquist-stability-criterion-based SSR explanation for type-3 wind generators," IEEE Trans. Energy Convers., vol. 27, no. 3, pp. 807809, Sep. 2012.

[6] Z. Miao, "Impedance-model-based SSR analysis for type 3 wind generator and series-compensated network," IEEE Trans. Energy Convers., vol. 27, no. 4, pp. 984-991, Dec. 2012.

[7] — " "Impact of unbalance on electrical and torsional resonances in power electronic interfaced wind energy systems," IEEE Trans. Power Syst., vol. 28, no. 3, pp. 3105-3113, Aug. 2013

[8] J. Sun, "Impedance-based stability criterion for grid-connected inverters," IEEE Trans. Power Electron., vol. 26, no. 11, pp. 3075-3078, 2011.

[9] L. A. Pilotto, A. Bianco, W. F. Long, and A.-A. Edris, "Impact of tcsc control methodologies on subsynchronous oscillations," IEEE Trans. Power Del., vol. 18, no. 1, pp. 243-252, Jan. 2003.

[10] R. K. Varma, S. Auddy, and Y. Semsedini, "Mitigation of subsynchronous resonance in a series-compensated wind farm using FACTS controllers," IEEE Trans. Power Del., vol. 23, no. 3, pp. 1645-1654, Jul. 2008.

[11] D. Jovcic and G. N. Pillai, "Analytical modeling of TCSC dynamics," IEEE Trans. Power Del., vol. 20, no. 2, pp. 1097-1104, Apr. 2005.

[12] X. Jiang and A. Gole, "A frequency scanning method for the identification of harmonic instabilities in hvdc systems," IEEE Trans. Power Del., vol. 10, no. 4, pp. 1875-1881, Nov. 1995.

[13] N. Johansson, L. Angquist, and H.-P. Nee, "A comparison of different frequency scanning methods for study of subsynchronous resonance," IEEE Trans. Power Syst., vol. 26, no. 1, pp. 356-363, Feb. 2011.

[14] R. Rajaraman, I. Dobson, R. H. Lasseter, and Y. Shern, "Computing the damping of subsynchronous oscillations due to a thyristor controlled series capacitor," IEEE Trans. Power Del., vol. 11, no. 2, pp. 11201127, Apr. 1996.

[15] S. G. Jalali, R. A. Hedin, M. Pereira, and K. Sadek, "A stability model for the advanced series compensator (ASC)," IEEE Trans. Power Del., vol. 11, no. 2, pp. 1128-1137, Apr. 1996.

[16] R. Lasseter, S. Jalali, and I. Dobson, "Dynamic response of a thyristor controlled switched capacitor," IEEE Trans. Power Delivery, vol. 9, pp. $1609-1615,1994$.

[17] H. Othman and L. Angquist, "Analytical modeling of thyristor-controlled series capacitors for ssr studies," IEEE Trans. Power Syst., vol. 11, no. 1, pp. 119-127, 1996.

[18] A. Ghosh and G. Ledwich, "Modelling and control of thyristorcontrolled series compensators," IEE Proceedings-Generation, Transmission and Distribution, vol. 142, no. 3, pp. 297-304, 1995.

[19] S. R. Joshi and A. Kulkarni, "Analysis of ssr performance of tesc control schemes using a modular high bandwidth discrete-time dynamic model," Power Systems, IEEE Transactions on, vol. 24, no. 2, pp. 840-848, May 2009.

[20] S. R. Joshi, E. Cheriyan, and A. Kulkarni, "Output feedback ssr damping controller design based on modular discrete-time dynamic model of tcsc," Generation, Transmission Distribution, IET, vol. 3, no. 6, pp. 561573, June 2009.

[21] K. Kabiri, S. Henschel, J. Marti, and H. Dommel, "A discrete statespace model for ssr stabilizing controller design for tcsc compensated systems," Power Delivery, IEEE Transactions on, vol. 20, no. 1, pp. 466-474, Jan 2005.

[22] P. Mattavelli, G. C. Verghese, and A. M. Stankovic, "Phasor dynamics of thyristor-controlled series capacitor systems," IEEE Trans. Power Syst., vol. 12, no. 3, pp. 1259-1267, Aug. 1997.

[23] N. G. Hingorani and L. Gyugyi, Understanding FACTS: concepts and technology of flexible AC transmission systems. IEEE press New York, 2000.

[24] M. Belkhayat, "Stability criteria for ac power systems with regulated loads," Ph.D. dissertation, Purdue University, West Lafayette, 1997.

[25] M. Céspedes and J. Sun, "Modeling and mitigation of harmonic resonance between wind turbines and the grid," in Energy Conversion Congress and Exposition (ECCE). IEEE, 2011, pp. 2109-2116.

[26] B. Friedland, Control System Design: An Introduction to State-Space Methods. Courier Dover Publications, 2005.

[27] N. Lehtomaki, N. R. Sandell, and M. Athans, "Robustness results in linear-quadratic gaussian based multivariable control designs," Automatic Control, IEEE Transactions on, vol. 26, no. 1, pp. 75-93, 1981.

[28] IEEE SSR Working Group, "Second benchmark model for computer simulation of subsynchronous resonance," IEEE Trans. Power App. Syst., vol. 104, no. 5, pp. 1057-1066, 1985.

Lakshan Piyasinghe is a Ph.D. student at University of South Florida (USF). $\mathrm{He}$ received his Bachelor degree in Electrical Engineering in 2006 from University of Moratuwa, Sri Lanka. He started his Ph.D. study at USF in Fall 2010 and his research interests include dynamic modeling and analysis of power electronic systems and power systems.

Zhixin Miao (S'00 M'03 SM'09) received the B.S.E.E. degree from the Huazhong University of Science and Technology,Wuhan, China, in 1992, the M.S.E.E. degree from the Graduate School, Nanjing Automation Research Institute (Nanjing, China) in 1997, and the Ph.D. degree in electrical engineering from West Virginia University, Morgantown, in 2002.

Currently, he is with the University of South Florida (USF), Tampa. Prior to joining USF in 2009, he was with the Transmission Asset Management Department with Midwest ISO, St. Paul, MN, from 2002 to 2009. His research interests include power system stability, microgrid, and renewable energy.

Javad Khazaei is a Ph.D student at University of South Florida (USF). He received his Bachelor degree in Electrical Engineering from Mazandaran University (2009) and Master degree from Urmia University (2011) in Iran. He started his Ph.D study at USF in Summer 2013 and his research interests include Smart Grid modeling, Renewable Energy Integration, and Power Electronics.

Lingling Fan received the B.S. and M.S. degrees in electrical engineering from Southeast University, Nanjing, China, in 1994 and 1997, respectively, and the Ph.D. degree in electrical engineering from West Virginia University, Morgantown, in 2001.

Currently, she is an Associate Professor with the University of South Florida, Tampa, where she has been since 2009. She was a Senior Engineer in the Transmission Asset Management Department, Midwest ISO, St. Paul, MN, form 2001 to 2007, and an Assistant Professor with North Dakota State University, Fargo, from 2007 to 2009. Her research interests include power systems and power electronics. Dr. Fan serves as a technical program committee chair for IEEE Power System Dynamic Performance Committee and an editor for IEEE Trans. Sustainable Energy. 